\title{
Mantle convection in super-Earths
}

Mantle convection - the cycling of heat and material in the silicate interiors of planets - dictates many planetary characteristics, from planetary cooling and magnetism to atmospheric composition and habitability. Characterizing the relationship between mantle convection and planetary mass is a key challenge for understanding rocky exoplanets.

So far, equation-of-state models based on extrapolation from conditions in Earth's mantle have ignored mineral phase transitions at pressures expected in superEarths. To overcome this limitation, Arie van den Berg and colleagues developed a new material model based on ab initio calculations of mineral properties. They simulated convection in planets up to 20 Earth masses and identified three convective regimes: below 4 Earth masses, vigorous convection occurs throughout the mantle; from 4 to 12 Earth masses, convection is slow and concentrated in a shallow, mobile zone; and above 12 Earth masses, a mid-mantle layer of high viscosity separates two vigorous-convection zones. In the lower zone, perovskite $\left(\mathrm{MgSiO}_{3}\right)$ breaks down to $\mathrm{Mg}$ and Si oxides, leading to a low viscosity layer above the core-mantle boundary, characterized by small-scale convective structures (see the image).

van den Berg et al. suggest that further research into high-pressure phase changes and lower mantle convective regimes could reveal whether super-Earths have core dynamos capable of generating magnetic fields.

Tom Henshall

ORIGINAL ARTICLE van den Berg, A. P. et al. Mass-dependent dynamics of terrestrial exoplanets using ab initio mineral properties. Icarus. 317, 412-426 (2019)

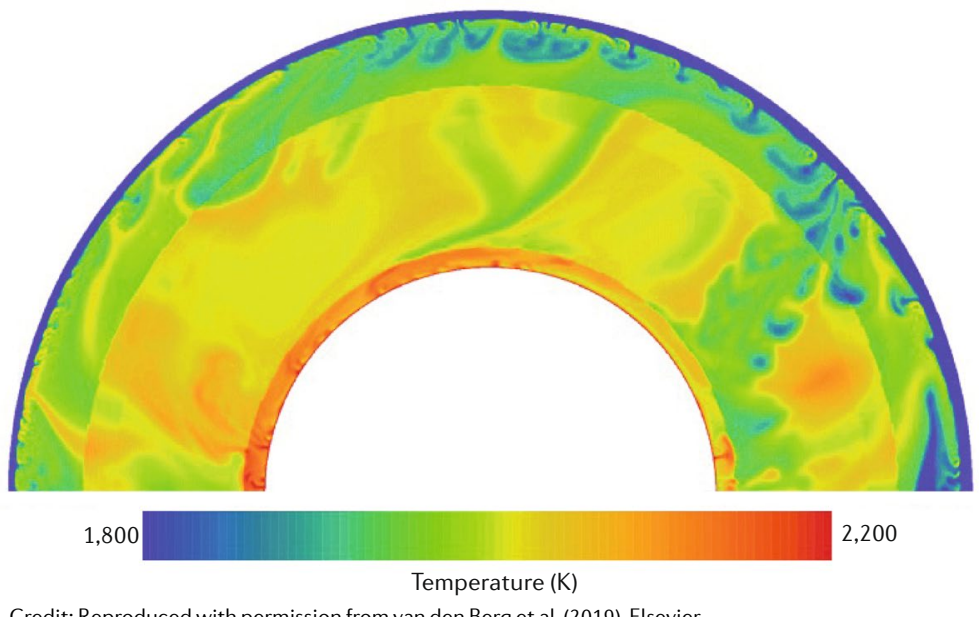

Credit: Reproduced with permission from van den Berg et al. (2019), Elsevier 